\title{
STUDY ON CULTURE POSITIVITY AMONG SPUTUM SMEAR NEGATIVE TUBERCULOSIS SUSPECTS ATTENDING THE NATIONAL TUBERCULOSIS CENTRE, NEPAL
}

\author{
Bichha RP'1, Karki KB'1, Sultana R', Jha KK'1, Salhotra VS'1, Khadka DK'1, Weerakoon AP' \\ ${ }^{1}$ SAARC Tuberculosis and HIVIAIDS Centre, Thimi, Kathmandu
}

\section{ABSTRACT}

Background: Globally, more than half of all TB cases are not detected. If they are not diagnosed and get treatment infection transmission may continue and patients suffer and may eventually die. Pulmonary TB either smear positive or negative is normally diagnosed by Ziehl- Neelsen stained sputum smear examination microscopy. Since the culture is the gold standard, evaluation of smear negative TB cases by this method is likely to detect more cases.

Objectives: The objective of this study is to find out culture positivity among smear negative patients.

Methods: All the three sputum samples reported negative by Ziehl-Neelsen microscopy from a total of 138 new TB suspected cases more than 15 years of age at NTC Laboratory. Nepal were cultured on Lowenstein-Jensen media. Tubes showing sufficient growth (culture positive) were recorded and all the culture positive results were informed to NTC. The culture positive samples were processed for DST on first line drugs using proportion method.

Results: The culture positivity rate was $5.1 \%$ (7 of 138 cases) All the positive cultures were processed for DST on first line anti-TB drugs and none showed resistance of the total 138 suspected Tuberculosis patients, $94(68.1 \%)$ were males and 44 were females (31.9\%) with male: female ratio 1:047. The mean age of the total patients were 30.69 . Nearly $42 \%$ of them belonged to $31-50$ years. Mean age of the male patients was significantly higher than total of the female $(p<.001)$.

Conclusions: Seven smear negative cases among the total of 138 suspected TB patients attending NTC were culture positive. Similar study has to be done in other parts of the country.

Key words: new TB suspects, smear negative, culture positive, diagnosis of tuberculosis.

\section{INTRODUCTION}

The detection and management of smear positive pulmonary disease is the principal aim of National Tuberculosis Control Programmes. However, smear negative disease is also a common clinical problem.

Several studies ${ }^{1}$ have identified the clinical characteristics of persons with the most infectious

\section{Correspondence:}

Mr. Dhruba Kumar Khadka

Consultant Microbiologist

Email: dkkhadka_888@yahoo.com, khadkadhruba8@gmail.com from of TB those harbouring the largest number of organisms, with acid-fast bacilli (AFB) found by microscopic examination of stained sputum (AFB smear positive). However, patients with active TB who have negative sputum smear results are also capable of transmitting the infection. (McPhredan FM et al/Grzybowski et al) ${ }^{2,3}$ The relative transmission rate of smear negative TB patients compared to smear positive TB patients has been calculated at $22 \%$ using a molecular epidemiological technique ${ }^{4}$. Although persons with smear negative TB are less infectious than the smear positive patients, the overall contribution to disease transmission is considerable because half of all patients with TB can present with negative sputum smear findings. ${ }^{5}$ 
The microscopic examination of sputum smear stained by Ziehl-Neelsen method can detect bacilli when they are of the order of $10^{4}$ per $\mathrm{ml}$ of sputum. Sputum smear microscopy has been shown to have a sensitivity of $96.5 \%$ specificity of $34.9 \%$ with a positive predictive valve of $17.3 \%$ and negative predictive value of $99.9 \%$ in Gizybowski's series. ${ }^{6}$

It is known that considerable transmission occurs before the level of bacilli reach $10^{4} \mathrm{ml}$ in the sputum. During this period of unknown duration the person continues to transmit the infection. It has also been established that sputum smear microscopy is less sensitive in HIV associated TB. Therefore it is important to diagnose from the smear-positive case of the future, not only for individual person but also for public health perspective. .

It has been proved that the probability of finding acid-fast bacilli by sputum smear microscopy is directly related to the concentration of bacilli in the sputum. Thus, when $1 \mathrm{ml}$ of sputum contains fewer than 10,000 but more than 1,000 organisms per $\mathrm{ml}$, the chances decreases rapidly, the result being negative in about $96 \%$ of cases. ${ }^{7}$

All sources of error being eliminated, the concentration of bacilli is the sputum is determined largely by the type of tuberculosis lesion from which the bacilli originate. Thus, a cavity about $2 \mathrm{~cm}$ in diameter (opening into a bronchus) may contain some $100^{8}$ i.e., one hundred thousand times fewer. Sputum from patients with tuberculosis lung cavities, containing softened necrotic particles with enormous amount of bacilli, will thus usually be found positive by direct smear microscopy. In contrast, sputum from patients with nodular, encapsulate lesion discharging only small amounts of bacilli will almost invariably be negative by smear microscopy. Only by using refined laboratory techniques, such as culture, can small number of bacilli be demonstrated ${ }^{9}$.

About 5000-10,000 acid fast bacilli (AFB) per millilitre of sputum must be present for detection by smear, whereas culture required only 10 100 viable organisms. ${ }^{10}$ Hence, smear negative culture positive patients generally have minimal disease with low bacillary counts rather than far advanced cavitary TB with heavy bacillary burden. For example, a US study of HIV negative culture positive patients estimated that negative smears are obtained from $60 \%-80 \%$ of patients with minimal disease, from $30 \%-40 \%$ of cases with more extensive disease, but only $5 \%-10 \%$ of patients with extensive cavitary lesions. ${ }^{11}$

Study conducted by the NTI Bangalore revealed that $35 \%$ culture positivity among smear negative patients. Smear negative culture positive cases are also associated with mortality. Longitudinal surveys conducted between 1961 and 1963 among untreated TB patients in Bangalore District, South India found that the mortality rate during the first 18 months of follow-up was $34.7 \%$ for smear postive patients, $14.1 \%$ for smear negative culture- positive cases, and about $5 \%$ for smear negative culture negative cases diagnosed radio logically. ${ }^{12}$

The difficulty of demonstrating small number of bacilli regularly has been shown in studies described elsewhere $(1970)^{13},(1971)^{14},(1976)^{16}$. The burden of sputum smear negative tuberculosis in Nepal is not known. According to our knowledge, culture results among smear negative TB suspects were not studied in Nepal. Hence, the aim of this study was to find out culture postivity among smear negative patients.

\section{MATERIALS AND METHODS}

\section{Study Design: Prospective study}

Study Population: All the new suspected TB cases attending National TB Centre (NTC) Laboratory, Nepal for diagnosis.

Inclusion criteria: All the new TB suspects more than 15 years of age attending the NTC OPD Clinic/ diagnosed by all three sputum smear \& declared smear negative on microscopic examination in NTC Laboratory.

Exclusion criteria: Smear +ve and/or AFB detected after sample processing/inoculation suspected EP and contaminated samples while processing for culture.

\section{Study site: NTC OPD Clinic/NTC Laboratory.}

Sampling method: Consecutive or non randomized sampling method was applied. The details of the patients (name, age, sex, type of patient, sputum examination requested for) were collected from the Laboratory register. 
Sample size: 138 . The sample size was calculated by using the formula recommended by $\mathrm{WHO}^{16}$ as $n=z^{2}(p)(1-p)$

$$
\mathrm{d}^{2}
$$

Where $\mathrm{n}=$ sample size

$Z=1.96$

$p=$ anticipated prevalence \# $(10 \%)$

$d=$ absolute precision $(5 \%)$

While applying equation/formula; $n=(1.96)^{2}$ *

$(0.1) 1-0.1) /(0.05)^{2}$

$$
\begin{aligned}
& =3.84 *(0.1)(0.9) / 0.0025 \\
& =138
\end{aligned}
$$

\# (Anticipated prevalence of $10 \%$ for smear negativity was assumed since the smear positivity rate among new case is $10 \%$ which is very close to the rate of smear negative or the ratio of smear positive and smear negative 1:1).

Sputum smear negative samples from a total of 138 new TB suspects (3 sputum samples per suspect were pooled and counted as one sample) were processed for this study. The samples showing AFB positive after processing or contamination, were replaced (approximately 10-15\%) with new sample from new TB suspects. Record for the same was maintained and intimated to NTC, Nepal whenever required.

Data Collection: A separate laboratory register (an original source of data) was maintained for the details of suspects and smear or culture results.

Ethical issue: As secondary sample was used institutional permission was taken from National Tuberculosis Center (NTC), Nepal.

\section{Laboratory procedures:}

- The NTC Laboratory staffs were oriented for keeping all the samples until microscopic examination and to coordinate with SAARC TB Reference Laboratory (STRL) staff.

- All three fresh sputum samples (3-5 ml each) found negative after examination were collected.

- After centrifugation, inoculation was done and observed every week upto eight weeks.

- All the positive cultures were processed for DST on first line anti-TB drugs (SHRE*) using proportion method.
- All the information for culture positive samples was sent to NTC. The DST results were also sent to NTC after completion. The records for culture and DST were maintained.

$$
\begin{aligned}
& S=\text { Streptomycin } \\
& H=\text { Isoniazid } \\
& \mathrm{R}=\text { Fifampicin } \\
& \mathrm{E}=\text { Ethambutol }
\end{aligned}
$$

\section{RESULTS}

\section{Age and sex distribution of the study sample}

Among the total of 138 suspected tuberculosis patients, $94(6.1 \%)$ were males and 44 were females $(31.9 \%)$ with male: female ratio 1:0.47. The mean age of the total patient was 30.69 years. Nearly $42 \%$ of them belonged to $31-50$ years age group. Mean age of the male patients was significantly higher than that of the female $(p<001)$.

Table 1: Distribution of Smear negative and culture positive results (sex \& age wise)

\begin{tabular}{|l|c|c|c|c|}
\hline \multirow{2}{*}{$\begin{array}{l}\text { Smear } \\
\text { negative }\end{array}$} & \multicolumn{4}{|c|}{ Culture positive } \\
\cline { 2 - 5 } & $\begin{array}{c}\mathbf{1 5 - 3 0} \\
\text { yrs }\end{array}$ & $\begin{array}{c}\mathbf{3 1 - 5 0} \\
\text { yrs }\end{array}$ & $\begin{array}{c}\mathbf{> 5 0} \\
\text { yrs }\end{array}$ & Total (\%) \\
\hline Male & 2 & 2 & 2 & $6(4.35 \%)$ \\
\hline Female & - & 1 & - & $1(0.72 \%)$ \\
\hline Total & 2 & 3 & 2 & $7(5.07 \%)$ \\
\hline
\end{tabular}

One hundred and forty seven (147) sputum samples were received in the STRL. Five samples

\begin{tabular}{|c|c|c|c|c|}
\hline \multirow{5}{*}{ 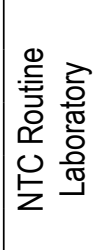 } & \multicolumn{4}{|c|}{ SAARC TB Reference Laboratory } \\
\hline & & Smear +ve & Smear-ve & Total \\
\hline & Smear +ve & 0 & 0 & 0 \\
\hline & Smear -ve & 4 & 140 & 144 \\
\hline & Total & 4 & 140 & 144 \\
\hline
\end{tabular}
were discarded due to the contamination. Another four samples were negative in the routine laboratory and positive in STRL were also excluded from the study.

Table 2: Comparison of smear results in reference laboratory and routine laboratory $(n=144)$

False negative rate at routine laboratory is $=4 / 144=2.8 \%$ 
Table 3: Culture positivity among smear negative TB suspects $(n=138)$

\begin{tabular}{|c|c|c|c|c|}
\hline \multirow{5}{*}{ 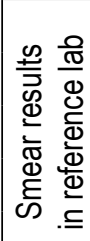 } & \multicolumn{4}{|c|}{ Culture results } \\
\hline & & Culture+ve & Culture-ve & Total \\
\hline & Smear +ve & 0 & 0 & 0 \\
\hline & Smear-ve & 7 & 131 & 138 \\
\hline & Total & 7 & 131 & 138 \\
\hline
\end{tabular}

Culture positivity rate among smear negative TB suspects $=7 / 138=5.1 \%$

DST pattern of culture positive patients $(n=7$ : No resistance was reported.

\section{DISCUSSION}

The most striking shortcoming perhaps of current TB control efforts is the inability of programmes to accurately diagnosed TB in a large proportion of patients, particularly in HIV infected individual and children. Globally, more than half of all TB cases are not detected, the results of health care system weakness and the inadequacy of available technology. If diagnosis is absent, patients are not treated, transmission may continue patients suffer needlessly and many eventually die. Pulmonary TB either smears positive or negative is normally diagnosed by Ziehl-Neelsen stained sputum smear microscopy. Major drawback of this method is, it requires more than 5000 bacilli per $\mathrm{ml}$ of sputum to detect on a smear. Since the culture is the gold standard, evaluation of smear negative TB cases by this methods is likely to detected more cases.

Before the HIV epidemic, two well- performed sputum examination were considered as a single culture. $^{12}$ With the increasing rate of smearnegative disease and the rising prevalence of drug resistance, culture facilities may need to be more widely available in developing countries, and new convenient TB diagnostics are urgently needed. ${ }^{17}$

During this study, we found that $7(5.1 \%)$ smear negative TB suspects were culture positive. Study conducted by Dhingra et al ${ }^{18}$ reported $9.3 \%$ of culture positivity among smear negative patients. Similarly, Prasad $^{19}$ reported $7 \%$ culture positivity among smear negative cases. In our study samples from TB suspects were taken where as in other 2 study compared to other studies may be due to this reason.
In a Romanian and Indian study, $35 \%$ of 557 Romanian patients and $28 \%$ of 296 Indian patients had positive cultures initially. ${ }^{20}$

The proportion of cases of smear-negative tuberculosis will depend primarily on clinical mix, but can be minimized by: (a) good sampling (b) careful technique (c) technical expertise (d) good microscopic facilities. There will still be a proportion of patients in whom organisms are scanty such patients pose a diagnostic challenge.

The association of AFB-negative smears with lower bacillary burdens and minimal pulmonary lesions would imply that the infectivity and the mortality of smear negative disease should be lower, and that less intensive chemotherapy may adequately treat this condition. Study conducted by Rouillon et al ${ }^{10}$ revealed that revealed that the prevalence of infection among children exposed to smearpositive cases varied between $30 \%-65 \%$, whereas the tuberculin reactivity rate was only $4.7 \%-26.8 \%$ among contacts of smear negative patients, whose TB diagnoses were based on positive culture. In Rouillion's study prevalence of infection was measured by tuberculin reactivity. However, in studies in high incidence environment in India and Africa, the infection rate among young contracts of smear-negative cases was similar to that in the general population or in households without documented TB. ${ }^{10}$ To prevent such transmission it necessary to detect culture positive cases.

The sensitivity of the AFB result is known to be poor, varying between $30 \%$ and $70 \%$ depending on a number of factors relating to how the test is implemented. The sensitivity is improved by concentration of sputum specimens and use of fluorescent microscopy, but reduced in patients with HIV disease.

Truly negative smear results of course, do not preclude TB disease. Culture of clinical specimens will confirm the diagnosis in smear positive cases and usually identify that many cases again of smear negative disease. This is because the culture is more sensitive ( $80 \%-85 \%)$, being able to detect as few as 10 bacteria per ml of sputum. It is also very specific (98\%) and allows, DST as well as genotyping of the isolate. ${ }^{21}$ Until recently, smear negative TB cases were thought not to contribute significantly to secondary transmission. The frequency of coughing, viscosity of the sputum, organism virulence and host factors are likely to play a role. Population based studies have estimated that smear negative patients with 
TB may contribute to at least $17 \%$ of the disease incidence in a community.

Patients with smear negative PTB are substantially less infectious than those with smear positive PTB. The risk of contracting disease for house hold contract of smear negative, culture positive patients is about one tenth of that for contracts of smear positive patients. Positive culture of $\mathrm{M}$. Tuberculosis with smear negative PTB in subSaharan Africa revealed that about one quarter to one third of the smear negative pulmonary patients were confirmed diagnosed by culture as tuberculosis. Most of the smear negative cases found to have tuberculosis by culture were also diagnosed as HIV positive serologically.

In Zimbabwe; 12 smear negative cases among 36 HIV +ve found to be culture positive for tuberculosis. Likewise, Zambia 11 cases of 27 HIV positive Rwanda 17 cases of 92 HIV positive and Malawi 30 cases of total 73; HIV test not done).

Children are more likely to become infected with TB and to be more susceptible to acquiring TB from smear negative sources. In one study done in British Columbia, Canada showed that $10 \%$ of children with TB acquired their disease from smear negative sources. During this study, $2.8 \%$ of false negative rate in routine laboratory was detected in comparison to reference laboratory. In many laboratories the staffs have to perform a wide range of duties, which reduces the time devoted to sputum smear microscopy.

Many positive AFB sputum specimens become falsely negative if left in a sputum container for over a week. There is often quality control performed, either by the laboratories themselves of by Central Reference Laboratory. The sensitivity of microscopic diagnosis could be improved by liquefying sputum with house hold bleach (sod hypochlorite sol) and concentrating mycobacteria by centrifugation. In Ethiopia, the sensitivity of sputum smears compared with culture was $31 \%$ when smear were prepared directly from sputum after sod hypo treatment and centrifugation. ${ }^{22}$

There are three types of laboratory services run by NTC Nepal (routine sputum examination, general laboratory, and culture and DST laboratory. Besides these, being a National TB Reference Laboratory, planning, procurement, training, supply, supervision, EQA of sputum microscopy for central region of the country as well as 5 regional quality control laboratories is done by 8 laboratory staff ( 2 Med technologists, 5 Lab technicians and one Lab attendant). NTC lab examines averagely 100 samples a day and smears are prepared directly from sputum (may fish up saliva only or miss the stuffy part). In this study, 3 smear negative samples were pooled and taken as one/ suspect, and homogenization or liquefaction of the specimen was done with $4 \% \mathrm{NaOH}$ followed by centrifugation to concentrate organisms. In such way, the accumulation of the organisms may have increased. But among a total of 4 false negative smears; 3 were scanty to $1+$ positive and only one was detected $3+$ by SAARC TB Reference Laboratory, for which sputum specimen were not collected on the same day. Nevertheless, each step of the laboratory procedure of sputum collection to recording and reporting should be completed properly, correctly and appropriately.

Till date, no study done for significant data how much is the culture positivity among smear negatives, in Nepal, It is encouraging that out of 7 culture positives none of the culture was resistant to first line Anti TB drugs.

\section{CONCLUSION AND RECOMMENDATION}

Seven smear negative cases among the total of 138 suspected TB patients attending NTC, were culture positive. Internal QC should be regulated in NTC laboratory and regular. Refresher training to laboratory staff is important. NTC laboratory should participate in national EQA programme and national EQA system should be strengthened. Similar survey to be done in other parts of the country, where high incidence and prevalence of TB cases occurs and the proportion of smear positive and negative is very close to minimize the chances of disease transmission especially to the children (0-14 yrs).

\section{CONFLICT OF INTEREST}

None

\section{REFERENCES}

1. Bock NN, McGowan JE, Jr. Ahn J, et al (1996) Clinical predictors of tuberculosis as a guide of a respiratory isolation policy. Am J Respir Crit Care Med 154, 1468-1472

2. Grzybowski S, Barnett GD, Stybol K (1075) Contracts of cases of active pulmonary tuberculosis. Bull Int. Union Tuberc 50; 90-106 
3. Behr MA, Warren SA, Salamon Hetal. Transmission of Mycobacterium tuberculosis from patients smear negative for acid fast bacilli [published erratum appears in Lancet 1999,353:1714]. Lancet 1999; $353 ; 444-449$

4. McPhredran FM, Opie EL. The spread of tuberculosis in families. AM J Hygiene. 1935;22:55 $-643$

5. Dutt AK, Stead WW. Smear negative pulmonary tuberculosis (published erratum appears in Semin Respir Infect 1994:9:261] Semin Respir Infect 9,113-119

6. Toman K. Toman's Tuberculosis Case Detection, Treatment and Monitoring, WHO lind edition 2004, p 12. http;//books.google.com.np/books http;// booksorders.who.int/booksorder/against/detart1. jsp

7. Grzybowski S, Harriett GD, Slyblo K. Contracts of cases of active pulmonary tuberculosis. Royal Netherlands Tuberculosis Association Selected Papers, 1975, 90

8. Canetti. G. The tubercle bacillus in the pulmonary lesion. New York, Springer, 1955. p 180.

9. Adler $H$. And Toman K. Acute tuberculosea Scandinavia. 25:178 (1950)

10. Rouillion A, Perdizet S, Parrpt R. Transmission of tubercle bacilli: the effect of chemotherapy. Tubercle 1976:57:275 -299.

11. Kim TC Blackman RS. Heatwole KM. Acid Fast Bacilli in sputum smears of patients with pulmonary tuberculosis. Am Rev. Respir Dis 1984;129:264 $-368$

12. Editorial. Smear negative pulmonary tuberculosis. Tubercle 1980:61:113-115

13. Nagapaul, D, R et al. Bulletin of World Health Organization 43:17 (1970).
14. Nair S. S. et. Al. Indian Journal of Tuberculosis 23; 152 (1976)

15. Van Genus HA, Contract study, Rotterdam. The Hague, IUAT Tuberculosis Surveillance Research Unit 1971.

16. Lwanga SK and Lemeshow S. Sample size determination in Health Studies. A Practical Manual. WHO, 1991.

17. Foulds J, O'Brien R. New tools for the diagnosis of tuberculosis; prospective of developing countries. Int J Tuberc Lung Dis 1998;88: 778-783.

18. Dhingra VK et al. Validity and reliability of sputum smear examination as diagnostic and screening test of tuberculosis. Indian J Allergy Asthma Immunol 2003: 17 (2): 67-69.

19. Prasad M, BAL A, Bhadur P. Laboratory Diagnosis of Pulmonary tuberculosis. Ind. J. Tub.; 58 (1):2226

20. S. L. Chan, Wanchai. Outcome of untreated smear negative pulmonary tuberculosis Symposia abstract; Tubercle and Lung Disease 1996; 2. In; The Dilemma of Smear Negative Pulmonary tuberculosis

21. R. K. Elwood, V.J. Cook and Hernandez- Garduno. Risk of tuberculosis in children from smear negative source cases; Int J tuberc Lung Dis. 2005; 9 (1):4955 .

22. Harries A.D., Mahler D. and Nunn P. An approach to the problems of diagnosing and treating adults smear negative pulmonary tuberculosis in high HIV-prevalence setting in sub-Saharan Africa 1998; In Bulletin of World Health Organization. 1998, 76 (6): 651-662.

23. Cobett EL, Walker N, Maher D, et al. The growing burden of tuberculosis. Global trends and interactions with the HIV epidemic. Arch Intern Med 2003; 163: 1009-1021. 